Case Report

\title{
Immunohistochemical and Ultrastructural Analyses of Cytoplasmic Blood Plasma Inclusions of Rat Hepatocytes
}

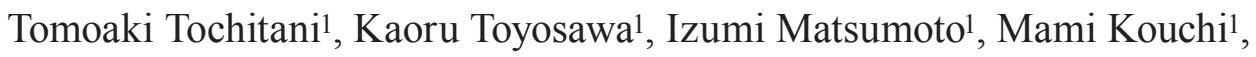 \\ Yoshiko Michimae', Takatoshi Koujitani ${ }^{1}$, Hitoshi Funabashi1, and Takaki Seki ${ }^{1}$ \\ ${ }^{1}$ Safety Research Laboratories, Dainippon Sumitomo Pharma Co., Ltd., 3-1-98 Kasugade-naka, Konohana-ku, Osaka \\ 554-0022, Japan
}

\begin{abstract}
In the present study, we investigated the histological, immunohistochemical and ultrastructural characteristics of cytoplasmic blood plasma inclusions that spontaneously occurred in a rat liver. Histologically, a number of cytoplasmic inclusions were observed in the liver of an 8-week-old female SD rat. These inclusions were strongly positive for PAS staining and resistant to diastase digestion. Immunohistochemical analyses revealed that these inclusions were positive for albumin and IgG; however, most of them were negative for LAMP-1 and LAMP-2. Ultrastructurally, the inclusions were surrounded by limiting membranes and composed of moderately electron dense, homogenous materials. These characteristics described here represent valuable information for pathological examination in toxicity studies. (DOI: 10.1293/tox.24.245; J Toxicol Pathol 2011; 24: 245-249)
\end{abstract}

Key words: rats, liver, spontaneous lesions, blood plasma inclusions

Blood plasma inclusions, resulting from accumulation of blood plasma in cells, are known to occur spontaneously in the canine liver1. Also, they can be induced in the rat liver by partial hepatectomy or by parental administration of albumin, serum, whole blood and other materials ${ }^{2,9}$. However, detailed investigations of these inclusions have rarely been reported, especially in the rat. In the present study, we investigated the histological, immunohistochemical and ultrastructural characteristics of cytoplasmic blood plasma inclusions that spontaneously occurred in a rat liver.

A female Crl:CD (SD) rat purchased from Charles River Laboratories Japan, Inc. (Kanagawa, Japan) was assigned to the middle dose group of a 2-week toxicity study and euthanized by exsanguination under isoflurane anesthesia at 8 weeks of age. Procedures for animal care and housing were in compliance with the institutional guidelines for the care and use of laboratory animals. Though no abnormalities were observed in general conditions, body weight, food consumption, hematology or at necropsy, a blood biochemical test revealed a slight elevation in AST (161 U/L). The

Received: 27 April 2011, Accepted: 4 August 2011

Mailing address: Tomoaki Tochitani, Dainippon Sumitomo Pharma Co., Ltd., 3-1-98 Kasugade-naka, Konohana-ku, Osaka 554-0022, Japan

TEL: 81-6-6466-5366 FAX: 81-6-6466-5446

E-mail: tomoaki-tochitani@ds-pharma.co.jp

(C2011 The Japanese Society of Toxicologic Pathology

This is an open-access article distributed under the terms of the Creative Commons Attribution Non-Commercial No Derivatives (by-ncnd) License $<$ http://creativecommons.org/licenses/by-nc-nd/3.0/>. present change was judged to be spontaneous because it was observed only in this animal and there was no doseresponse relationship.

For routine histopathological examination, most systemic organs and tissues were fixed in $10 \%$ neutral buffered formalin, and paraffin-embedded sections were prepared and stained with hematoxylin and eosin (H\&E). Sections of the liver were also stained by the Oil red-O method, Masson's trichrome method and periodic acid-Schiff (PAS) method with or without diastase digestion. For the Oil red-O method, frozen sections of the liver were used. For immunohistochemical examination, sections of the liver were subjected to a labeled polymer method using Histofine Simple Stain Rat MAX-PO (MULTI) (Nichirei Biosciences Inc., Tokyo, Japan). Table 1 shows the primary antibodies used in this study. For the normal and phospholipidosis-affected rat liver samples, archival paraffin-embedded tissue blocks were utilized from another toxicity study. Phospholipidosis was confirmed ultrastructurally. For electron microscopic examination, small pieces of the liver fixed in neutral buffered $10 \%$ formalin were refixed in $2.5 \%$ glutaraldehyde, postfixed in $1 \%$ osmium tetroxide and routinely embedded in Epon resin. Ultrathin sections of the selected areas were prepared, contrasted with hafnium chloride and lead citrate and examined using a Hitachi 7600 transmission electron microscope (Hitachi High-Technologies Corporation, Tokyo, Japan).

Histologically, a number of cytoplasmic inclusions were found in the hepatocytes mainly in the centrilobular zone of the subcapsular area (Fig. 1A). In addition, cytoplas- 
Table 1. Antibodies Used in This Study

\begin{tabular}{lccccl}
\hline Antibody & Product number & Antigen species & Dilution & Antigen retrieval a & Source ${ }^{\mathrm{b}}$ \\
\hline Albumin & A0001 & Human & $1: 1000$ & Heat & Dako \\
IgG & ab6703 & Rat & $1: 200$ & Proteinase K & Abcam \\
LAMP-1 & ab24170 & Human & $1: 200$ & Heat & Abcam \\
LAMP-2 & L0668 & Mouse & $1: 100$ & Heat & Sigma-Aldrich \\
\hline
\end{tabular}

${ }^{a}$ Heat: pressure cooker/citrate buffer ( $\mathrm{pH}$ 6.0), 10 minutes. Proteinase K: $0.4 \mathrm{mg} / \mathrm{mL}$, room temperature, 5

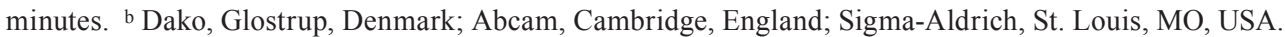

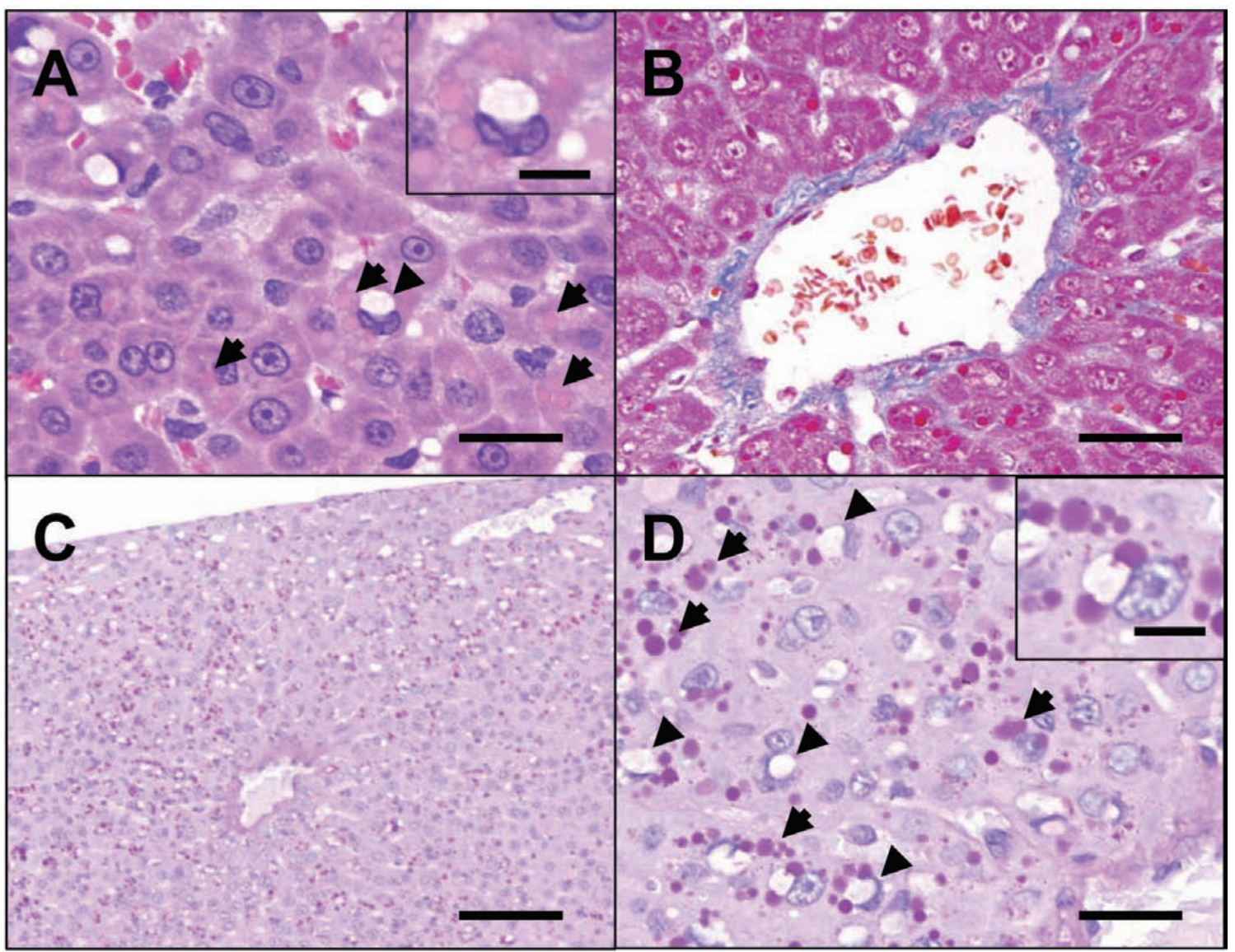

Fig. 1. Histological findings of the liver. A number of cytoplasmic inclusions (arrows) and cytoplasmic vacuoles (arrowheads) were found in the hepatocytes (A). H.E. stain. Bar=50 $\mu \mathrm{m}$. Inset: Higher magnification of a hepatocyte with inclusions and a vacuole. Bar=20 $\mu \mathrm{m}$. Also, slight centrilobular fibrosis was seen (B). Masson's trichrome staining. Bar $=70 \mu \mathrm{m}$. These inclusions (arrows) were strongly positive for PAS staining with diastase digestion and mainly seen in the centrilobular zone of the subcapsular area (C and D). On the other hand, the vacuoles (arrowheads) were negative for PAS staining (D). Bars $=100 \mu \mathrm{m}$ (C) and $50 \mu \mathrm{m}$ (D). Inset: Higher magnification of a hepatocyte with inclusions and a vacuole. $\mathrm{Bar}=20 \mu \mathrm{m}$.

mic vacuoles were seen in the same region. Slight centrilobular fibrosis was also observed (Fig. 1B). Inclusions were round, measuring less than $20 \mu \mathrm{m}$ in diameter, clearly delimited and stained homogenously eosinophilic. Generally, they were observed in a cluster and scattered in cytoplasm. They were strongly positive for PAS staining and resistant to diastase digestion (Figs. 1C, 1D) and were negative for Oil red O-staining (data not shown). On the other hand, the vacuoles were less than $20 \mu \mathrm{m}$ in diameter and observed solitarily in each hepatocyte. On average, the vacuoles were larger than inclusions. They were located adjacent to the nucleus and frequently invaginated into it. Also, the vacuoles were negative for both PAS staining and Oil red O-staining (Fig. 1D). No abnormality was observed in other tissues histopathologically examined.

Immunohistochemical analyses revealed that these inclusions were positive for albumin (Fig. 2A) and IgG (Fig. 2B). Most of the inclusions were negative for LAMP-1 (Fig. 2C) and LAMP-2 (Fig. 2D), though some were very weakly positive for LAMP-2. By contrast, phospholipidosis-affect- 


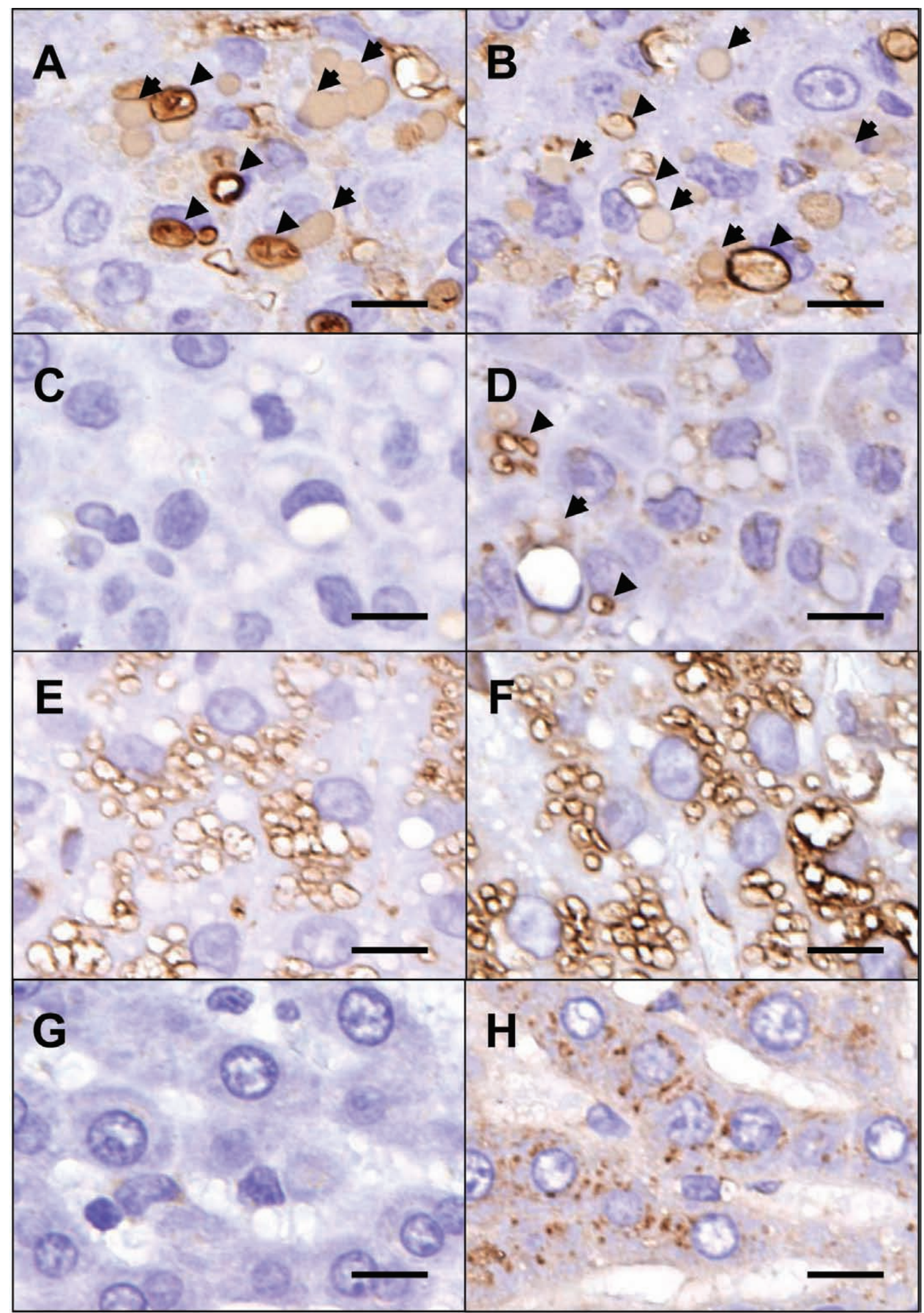

Fig. 2. Immunohistochemical findings of the liver. Inclusions (arrows) were positive for albumin (A) and $\operatorname{IgG}(\mathrm{B})$. Most of the inclusions were negative for LAMP-1 (C) and LAMP-2 (D), though some were very weakly positive for LAMP-2 (D, arrow). Some of the cytoplasmic vacuoles (A and B, arrowheads) were also positive for albumin and IgG. Also, some smaller vacuoles were positive for LAMP-2 (D, arrowheads). Phospholipidosis-affected rat hepatocytes, which were filled with phospholipid-containing lysosomes, were strongly positive for both LAMP-1 (E) and LAMP-2 (F). LAMP-1-positive granules were barely visible in the normal rat liver (G), while LAMP-2-positive granules could be seen even in normal hepatocytes $(\mathrm{H})$. Bars $=25 \mu \mathrm{m}$. 


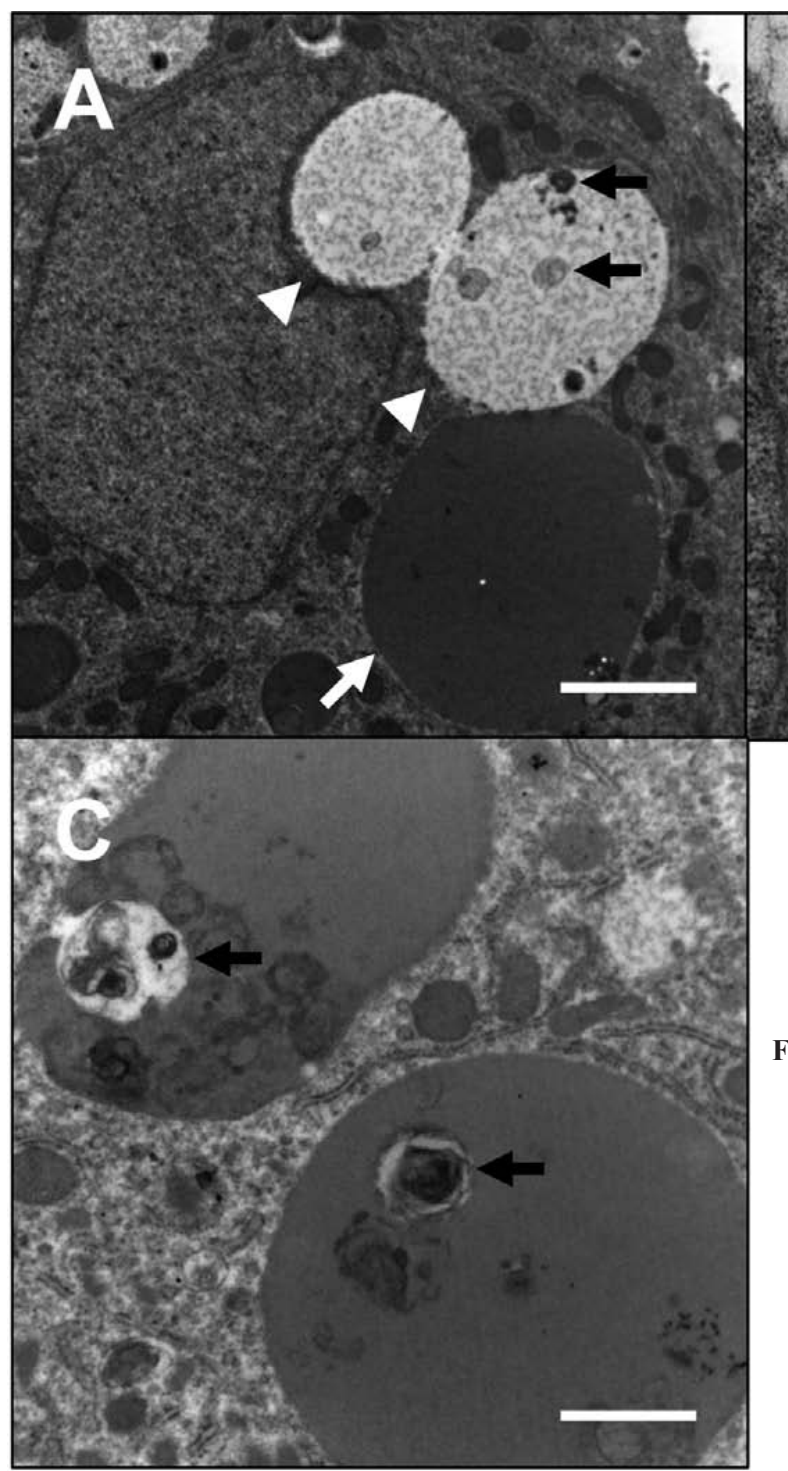

Fig. 3. Ultrastructural findings. Gross appearance of a hepatocyte with inclusion and vacuoles (A) and higher magnification (B). Vacuoles were located adjacent to the nucleus and invaginated into it. The inclusions (white arrows) were surrounded by limiting membranes and composed of moderately electron dense, homogenous materials. On the other hand, the vacuoles (arrowheads) did not have obvious membranes and were filled with electron lucent, flocculent materials. Some vacuoles (A) and inclusions (C) contained debris of unknown origin (black arrows). Bars $=2 \mu \mathrm{m}(\mathrm{A}), 0.5 \mu \mathrm{m}(\mathrm{B})$ and $1 \mu \mathrm{m}(\mathrm{C})$.

ed rat hepatocytes, which were filled with phospholipidcontaining lysosomes and used as a positive control, were strongly positive for both LAMP-1 (Fig. 2E) and LAMP-2 (Fig. 2F). LAMP-1-positive granules were barely visible in the normal rat liver (Fig. 2G), while LAMP-2-positive granules could be seen even in normal hepatocytes (Fig. 2H). Some of the cytoplasmic vacuoles were also positive for albumin and IgG (Figs. 2A and 2B). Moreover, some smaller vacuoles were positive for LAMP-2 (Fig. 2D).

Ultrastructurally, the inclusions were surrounded by limiting membranes and composed of moderately electron dense, homogenous materials (Fig. 3). On the other hand, the vacuoles did not have obvious membranes and were filled with electron lucent, flocculent materials. Also, some inclusions and vacuoles contained debris of unknown origin (Figs. 3A, 3C). Thus, these inclusions were judged to be intracytoplasmic blood plasma inclusions.

In the rat liver, other structures such as mallory bodies and giant mitochondria are also observed as eosinophilic cytoplasmic inclusions. Mallory bodies are suggested to result from condensation or collapse of intermediate filaments ${ }^{4}$, and ultrastructurally they are composed of fibrous materials $^{3}$, unlike the present case. Giant mitochondria are negative for PAS staining, and their double membranes and cristae can be observed in ultrastructural analysis ${ }^{8}$. Moreover, none of the common rat viruses are associated with hepatocytic intracytoplasmic inclusions 5 .

The histological aspects of blood plasma inclusions in this study were comparable to those of a published case ${ }^{1}$. Also, as previously reported 2,9 , the inclusions were strongly positive for PAS staining with diastase digestion and negative for Oil-red $\mathrm{O}$, and immunohistochemistry revealed that they contained a blood plasma component. Blood plasma inclusions are thought to be caused by disturbance in blood flow, such as an increase in intrasinusoidal pressure ${ }^{1}$. In the present case, though the precise mechanism of formation of inclusions was uncertain, the subcapsular distribution of inclusions and finding of centrilobular fibrosis suggested that 
they also occurred in a context of poor peripheral circulation? ${ }^{7}$ Also, the mechanism of AST elevation observed in this animal was not clear.

In this study, cytoplasmic vacuoles that were free of fat or glycogen were observed adjacent to inclusions. Similar vacuoles have been reported in the rat liver and have been suggested to be caused by anoxia ${ }^{10}$. Considering that these vacuoles also contained a blood plasma component, it is possible that they were formed by the same mechanism as the inclusions. However, the relationship between these two structures was uncertain, since the transition between them was not clearly seen.

Anderson et al. suggested a relationship between blood plasma inclusions and lysosomes on the basis of their histochemical reaction to acid phosphatase ${ }^{2}$, but we could not elucidate this possibility because most inclusions in the present study were negative for LAMP-1 and LAMP-2, novel lysosome markers ${ }^{6}$. However, the ultrastractural characteristics of the inclusions were similar to those of lysosomes, and some of the inclusions and vacuoles were positive for LAMP-2, suggesting some involvement of lysosomes. So, further research will be needed to clarify the role of lysosomes in the development of these inclusions and vacuoles.

Here, we described the detailed characteristics of blood plasma inclusions that spontaneously occurred in a rat liver. This will provide valuable information for pathological examination in toxicity studies.

Acknowledgements: We wish to thank the technicians in the pathology laboratory for carrying out the histotechnical work.

\section{References}

1. Abe K, Shimoda T, and Shikata T. Cytoplasmic blood plasma inclusions of canine hepatocytes demonstration by immunoperoxidase-labeling method. Am J Vet Res. 41: 1507-1511. 1980. [Medline]

2. Anderson PJ, Cohen S, and Barka T. Hepatic injury. A histochemical study of intracytoplasmic globules occurring in liver injury. Arch Pathol. 71: 89-95. 1961. [Medline]

3. Denk H, Eckerstorfer R, Gschnait F, Konrad K, and Wolff K. Experimental induction of hepatocellular hyalin (Mallory bodies) in mice by griseofulvin treatment. 1. Light microscopic observation. Lab Invest. 35: 377-382. 1976. [Medline]

4. French SW. The Mallory body: structure, composition, and pathogenesis. Hepatology. 1: 76-83. 1981. [Medline] [CrossRef]

5. Jacoby RO, Bhatt PN, and Jonas AM. Viral diseases. In: The Laboratory Rat, vol I, Biology and Diseases. HJ Baker, JR Lindsey, and SH Weisbroth (eds). Academic, New York. 271-306. 1979.

6. Obert LA, Sobocinski GP, Bobrowski WF, Metz AL, Rolsma MD, Altrogge DM, and Dunstan RW. An immunohistochemical approach to differentiate hepatic lipidosis from hepatic phospholipidosis in rats. Toxicol Pathol. 35: 728-734. 2007. [Medline] [CrossRef]

7. Parker GA, and Gibson WB. Liver lesions in rats associated with wrapping of the torso. Toxicol Pathol. 23: 507-512. 1995. [Medline] [CrossRef]

8. Sano T, Ozaki K, Matsuura T, and Narama I. Giant mitochondria in pancreatic acinar cells of alloxan-induced diabetic rats. Toxicol Pathol. 38: 658-665. 2010. [Medline] [CrossRef]

9. Schlicht I. Experimental studies on hyaline-drop protein uptake by the liver. Virchows Arch Pathol Anat Physiol Klin Med. 336: 342-353. 1963. [Medline] [CrossRef]

10. Trowell OA. The experimental production of watery production of the liver. J Physiol. 105: 268-297. 1946. 